\title{
ChemComm
}

\section{Poly(3-alkylthiophene)s show unexpected second-order nonlinear optical response $\dagger$}

Cite this: Chem. Commun., 2014 50,2741

Received 22nd October 2013, Accepted 16th January 2014

DOI: $10.1039 / c 3 c c 48099 b$

www.rsc.org/chemcomm

Regioregular poly(3-hexylthiophene)s with chain lengths varying from 5 to $\mathbf{1 0 0}$ monomers are synthesized. Poly(3-hexylthiophene)s show in solution an unexpectedly significant second-order nonlinear optical response. The increase in transition dipole moment upon oligomerisation causes the significant second-order nonlinear optical response.

Second-order nonlinear optical materials are applied as electrooptical modulators, for frequency doubling, terahertz generation, ${ }^{1}$ and are more intensively investigated as materials for optical computing. ${ }^{2}$ So far, nearly all commercially used materials are inorganic materials. Organic materials however show faster response times, ${ }^{3}$ and have more process flexibility, providing more potential for downscaling into functional devices. In this context, active polymers are of particular interest due to their increased stability over functionalised blends.

The versatile use of poly(3-alkylthiophene)s and conjugated polymers in general is widely explored in research fields such as organic field effect transistors (OFET), solar cells, organic light emitting diodes (OLED) and nonlinear optical devices. ${ }^{4}$ The broad applicability of polythiophenes resulted in a large product diversity as well as in a wide range of synthetic routes to produce them in reasonable yields and obtain meso and nano scale architectures. ${ }^{5}$ Although already investigated for their third order nonlinear optical response, ${ }^{6}$ their second order nonlinear optical response has not been investigated thoroughly. This is largely due to the fact that the structure of polythiophenes does not follow the typical donor conjugated structure - acceptor paradigm, and therefore no

\footnotetext{
${ }^{a}$ Laboratory for Molecular Electronics and Photonics, University of Leuven, Celestijnenlaan 200D, 3001 Leuven, Belgium

${ }^{b}$ Laboratory for Polymer Synthesis, University of Leuven, Celestijnen 200F, 3001 Leuven, Belgium

${ }^{c}$ Imec, Kapeldreef 75, 3001 Leuven, Belgium

${ }^{d}$ Catalysis Engineering, Chemical Engineering Department, Delft University of Technology, Julianalaan 136, BL 2826 Delft, The Netherlands.

E-mail: m.a.vanderveen@tudelft.nl

$\dagger$ Electronic supplementary information (ESI) available: Synthesis of P3HT, details of UV-VIS and HRS set-up and measurements and homogeneously damped two-level model. See DOI: 10.1039/c3cc48099b
}

significant second-order nonlinear effect is expected. Typically, high second-order nonlinear optical responses of materials are expected in extended conjugated systems that are non-centrosymmetrically organized. ${ }^{7}$ The dominant strategy is to utilize dipolar chromophores, either in the polymer backbone covalently bonded to the side chains or via doping polymers with chromophores. ${ }^{8}$

Previously, a scaling study of the second-order nonlinear optical response has been conducted on non-conjugated oligonucleotides up to 50 monomers. ${ }^{9}$ However, to the best of our knowledge, scaling studies of conjugated polymers are limited to $4-5$ monomers. ${ }^{10}$ Herein, we report for the first time the second-order nonlinear optical response of conjugated polymers upon increasing the chain length to 100 units. More specifically, regioregular poly(3-hexylthiophene)s (P3HT) are studied. Despite the limited donor strength of the alkyl substituents, an appreciably large second-order nonlinear optical response is observed. Upon increasing the polymerization degree the materials show a dramatic increase in the second-order response due to an increase in the transition dipole moment.

A series of varying chain lengths of regioregular head-to-tail coupled P3HT polymers (see Scheme 1) is synthesized. The small polydispersities $(\sim 1.2)$ were confirmed by gel permeation chromatography. The degree of polymerization was determined by ${ }^{1} \mathrm{H}$ NMR by end-group quantification. ${ }^{11} \mathrm{We}$ obtained a series ranging from 5 to 100 repeating monomer units (see ESI $\dagger$ ).

The second-order nonlinear optical response of the polymers was determined in solution via a Hyper-Rayleigh Scattering (HRS) experiment at a fundamental input wavelength of $800 \mathrm{~nm}$. The incoherent scattered light at the second harmonic wavelength of $400 \mathrm{~nm}$ is

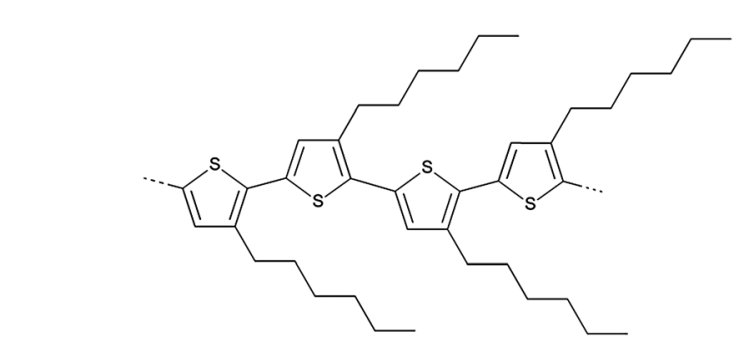

Scheme 1 Head-to-tail coupled regioregular poly(3-alkylthiophene). 
detected in a perpendicular geometry. To separate the frequency doubled light from two photon absorption fluorescence at the same frequency, we take advantage of the time-delay of fluorescence versus the quasi instant generation of frequency doubled light. This is done in the frequency domain according to the method described by Olbrechts et al. ${ }^{12}$ Further details of the experimental set-up and conditions can be found in the ESI. $\dagger$

We report the directly measured hyperpolarizability $\beta$, as well as the static hyperpolarizability $\beta_{0}$ in order to exclude contributions from resonance enhancement. The static hyperpolarizability $\beta_{0}$ is a frequency independent quantity that allows comparison of molecules that have different electronic resonances. It thus allows us to compare the performance of different organic molecules which is of relevance to optoelectronic applications. We thus adjust the redshift in electronic resonance frequency upon increasing the chain length of P3HT by applying the homogeneously damped two-state model to derive the static hyperpolarizability $\beta_{0}$ from the measured hyperpolarizability $\beta$ (see ESI $\dagger$ ). ${ }^{13}$

The P3HT oligomers/polymers are dissolved in a good solvent, chloroform, at a typical concentration of $0.1 \mathrm{mM}$ of monomer units to ensure complete solubility. In Fig. 1, we show the observed hyper-Rayleigh response per polymer chain $\beta_{\text {pol }}$ as a function of the number of monomer units per polymer chain $n$. An apparent linear increase of the HRS-response upon chain lengthening is observed (the fitted line is guidance to the eye). However, when rescaling the data towards the number of monomer units in the polymer backbone $\left(\beta_{\mathrm{pol}} / n\right)$, a totally different trend is observed (Fig. 2). The depiction of $\beta_{\text {pol }} / n$ is a means of comparing the amount of second-order nonlinear optical response generated by molecules of different molecular weight as it depicts the hyperpolarizability for the same density of material. Initially the HRS response $\beta_{\mathrm{pol}} / n$ increases upon chain lengthening, but saturation occurs for chain lengths longer than 15 repeating monomer units. The same trend is observed for the directly measured $\beta_{\mathrm{pol}} / n$ as well as for the static $\beta_{\mathrm{pol}, \mathrm{o}} / n$ (see Fig. 2). At this point, it is worthwhile mentioning that the monomer unit by itself has a very small hyperpolarizability that cannot be measured using our HRS set-up. This implies that the 2nd order NLO response does not originate from the 3-alkylthiophene monomer itself, but from a cooperative effect of the monomers within one oligomer-polymer chain such as the delocalized $\pi$-conjugated backbone.

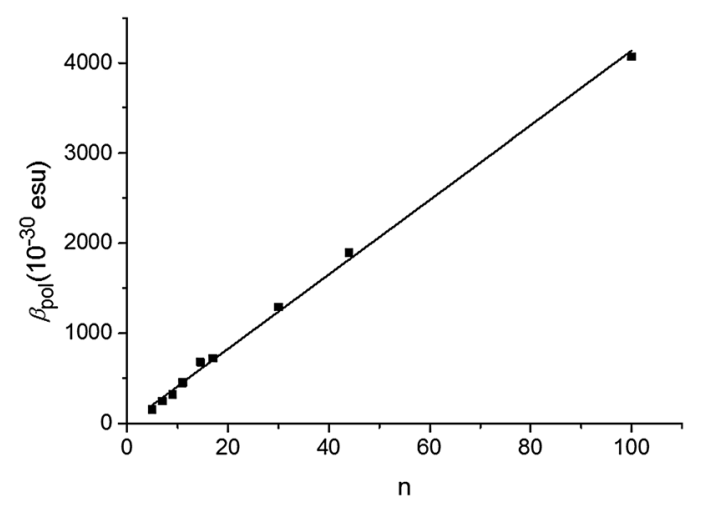

Fig. 1 Hyperpolarizability $\beta_{\text {pol }}$ versus the number of monomers $n$ of P3HT.

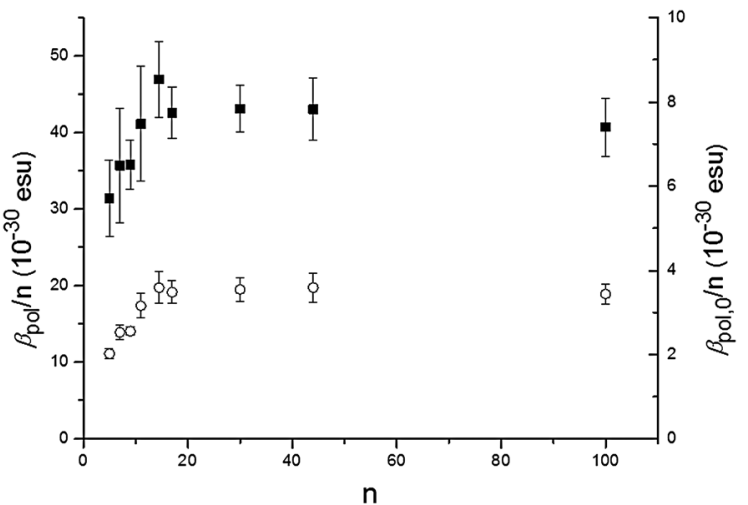

Fig. 2 Hyperpolarizability per monomer unit $\beta_{\text {pol }} / n$ (filled squares) and the static hyperpolarizability per monomer unit $\beta_{\mathrm{pol}, 0} / n$ (open circles) as a function of the number of monomer units $n$ of P3HT.

To understand which properties of molecules contribute to the second-order nonlinear response, we provide the description of the static hyperpolarizability $\beta_{0}$ within the two-state model where two states provide the dominant contribution to hyper-Rayleigh scattering $^{14}$

$$
\beta_{0} \sim\left(\mu_{\mathrm{ee}}-\mu_{\mathrm{gg}}\right)\left(\frac{\mu_{\mathrm{ge}}^{2}}{E_{\mathrm{eg}}^{2}}\right)
$$

with $\mu_{\mathrm{ee}}$ and $\mu_{\mathrm{gg}}$ being the dipole moment of the excited and ground states, respectively, $\mu_{\text {ge }}$ the transition dipole moment of the two states and $E_{\text {eg }}$ the energy difference between the two states. The used model assumes one transition between states to be dominant, consistent with the HOMO-LUMO transition in polythiophenes. The transition dipole moment and the transition energy difference can be obtained from the linear UV-VIS spectra of the polymer solutions (see ESI $\dagger$ ). From Fig. 3, we can see that the hyperpolarizability and transition dipole moment per monomer unit both saturate around 15 (or $1 / n=0.7$ ) monomer units, while the decrease in bandgap energy saturates only at much longer chain lengths. It is thus clear that the increase of the hyperpolarizability upon increasing the chain length follows the same trend as the increase of $\mu_{\mathrm{ge}}{ }^{2} / n$. It is clear from the data that the cooperative enhancement upon

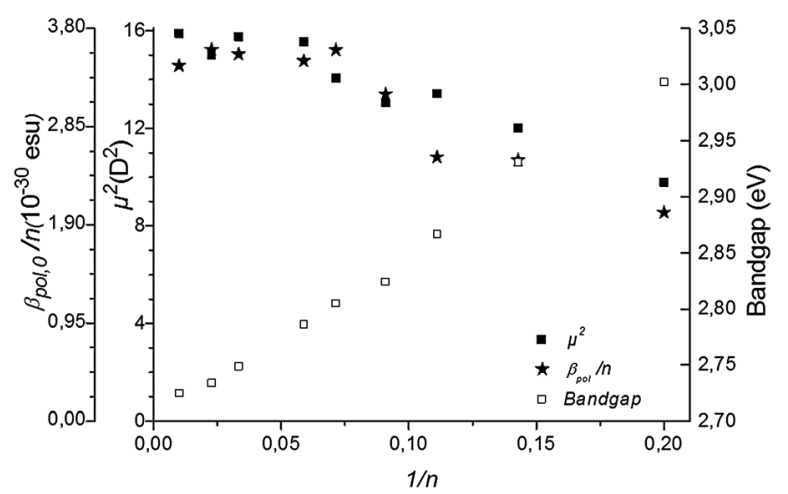

Fig. 3 The transition dipole moment $\mu_{\mathrm{ge}}{ }^{2}$ and the static hyperpolarizability per monomer unit $\beta_{\mathrm{pol}, 0} / n$ and the bandgap are given as a function of the inverse of the number of monomer units $n$ of P3HT. 
incorporation of hexylthiophene into the polymer is mainly due to the increase of conjugation.

Several effects can cause the non-centrosymmetry required for the second order NLO effect. The alkyl substituents, all in the 3-position in the regioregular P3HT, cause breaking of noncentrosymmetry (see Scheme 1). The effect on the NLO response is expected to be relatively small due to the limited donor strength of alkyl substituents. A non-planar conformation of the backbone $^{15}$ with helical structure can also cause asymmetry. As noncentrosymmetry is a requirement for the second-order NLO effect, the conformation will have an effect on the second order NLO response. Experimental and theoretical studies have shown that the persistence length - a measure of the length of rodlike or straight conformation within a polymer - of P3HT in a good solvent is about 10 units. $^{16}$ Yet, the effect of conformation is not clear from the data. Indeed, as can be seen from Fig. 2, the trend in the hyperpolarizability is largely explained by electronic effects.

As mentioned, the second-order nonlinear optical response of the monomer could not be measured. This means that the extension of conjugation leads to a dramatic enhancement of the second-order nonlinear optical properties of the material. This is also in line with the theoretical results that predict a generally larger than 3-fold increase of the hyperpolarizability upon extending from the thiophene monomer to the trimer. ${ }^{17}$ If we compare the static hyperpolarizability $\beta_{\text {pol,o }}$ for 5 units thus having $32 \ddagger$ conjugated electrons, $17.7 \pm 1.2 \times 10^{-30} \mathrm{esu}$, with $p$-nitroaniline, a benchmark NLO molecule, extended with two additional benzene rings to 4-nitro$4^{\prime \prime}$-amino- $p$-terphenyl, thus having 22 conjugated electrons, we find a very similar hyperpolarizability $\beta$, namely $16 \times 10^{-30}$ esu. $^{18}$ The second-order nonlinear optical response of $\mathrm{P} 3 \mathrm{HT}$ is thus very significant, while it was expected to be negligible. The best performing organic molecules typically have a hyperpolarizability one order of magnitude larger, ${ }^{19}$ but polythiophenes outperform these complex organic molecules with respect to easy synthesis and processability into devices. ${ }^{5,20}$ Moreover, in the solid-state polythiophenes display much more planar conformations with higher electron delocalization and less bending can be achieved. ${ }^{21}$ Based on the results reported here, we conclude that such solid-state polythiophenes could have an even larger second-order nonlinear optical response.

In conclusion, we found for oligomers of P3HT in solution that they, despite the limited donor strength of the substituents, show an unexpectedly significant second-order nonlinear optical response. This response is in the order of a benchmark nonlinear optical molecule. The dramatic increase in hyperpolarizability with chain length is largely attributed to the increased conjugation length. This is the first systematic study of second-order nonlinear scattering response of a conjugated polymer as a function of chain length.

MAvdV and SV acknowledge the Scientific Research Fund Flanders (FWO) for their personal fellowships. We are also grateful to the Onderzoeksfonds KU Leuven/Research Fund KU Leuven for a GOA and CREA grant.

\section{Notes and references}

$\$$ Each of the five thiophene units has 6 conjugated electrons. In addition, due to the synthesis procedure a bromo substituent is present at one end of the oligomer. This brings the total of conjugated electrons to 32 .
1 (a) L. R. Dalton, P. A. Sullivan and D. H. Bale, Chem. Rev., 2010, 110, 25-55; $(b)$ M. Lee, H. E. Katz, C. Erben, D. M. Gill, P. Gopalan, J. D. Heber and D. J. McGee, Science, 2002, 298, 1401-1403; (c) X. Zheng, C. V. McLaughlin, P. Cunningham and L. M. Hayden, J. Nanoelectron. Optoelectron., 2007, 2, 58-76; (d) L. R. Dalton, S. J. Benight, L. E. Johnson, D. B. Knorr, I. Kosilkin, B. E. Eichinger, B. H. Robinson, A. K.-Y. Jen and R. M. Overney, Chem. Mater., 2011, 23, 430-445.

2 J. L. O'Brien, Science, 2007, 318, 1567-1570.

3 J. M. Cole, Philos. Trans. R. Soc., A, 2003, 361, 2751-2770.

4 (a) G. Wang, J. Swensen, D. Moses and A. J. Heeger, J. Appl. Phys., 2003, 93, 6137; (b) G. Li, V. Shrotriya, J. Huang, Y. Yao, T. Moriarty, K. Emery and Y. Yang, Nat. Mater., 2005, 4, 864; (c) N. R. Armstrong, W. Wang, D. M. Alloway, D. Placencia, E. Ratcliff and M. Brumbach, Macromol. Rapid Commun., 2009, 30, 717-731.

5 (a) B. H. Cumpston, S. P. Ananthavel, S. Barlow, D. L. Dyer, J. E. Ehrlich, L. L. Erskine, A. A. Heikal, S. M. Kuebler, M. Rumi, X. Wu, S. R. Marder and J. W. Perry, Nature, 1999, 398, 51-54; (b) G. Zotti, B. Vercelli and A. Berlin, Acc. Chem. Res., 2008, 41, 1098-1109; (c) I. Osaka and R. D. McCullough, Acc. Chem. Res., 2008, 41, 1202-1214.

6 (a) S. Kishino, Y. Ueno, K. Ochiai, M. Rikukawa, K. Sanui, T. Kobayashi, H. Kunugita and K. Ema, Phys. Rev. B, 1998, 58, 430-433; (b) M.-T. Zhao, B. P. Singh and P. N. Prasad, J. Chem. Phys., 1988, 89, 5535; (c) Y. Verbandt, H. Thienpont, I. Veretennicoff, P. Geerlings and G. L. J. a. Rikken, Chem. Phys. Lett., 1997, 270, 471-475; (d) T. Bjornholm, D. R. Greve, T. Geisler, J. C. Petersen, M. Jayaraman and R. D. McCullough, Adv. Mater., 1996, 8, 920; (e) T. Bjornholm, D. R. Greve, T. Geisler, J. C. Petersen, M. Jayaraman and R. D. McCullough, Synth. Met., 1997, 84, 531-532.

7 T. Verbiest, K. Clays and V. Rodriguez, Second-Order Nonlinear Optical Characterization Techniques, CRC Press, Boca Raton, 2009.

8 (a) P. N. Prasad and D. J. Williams, Introduction to nonlinear optical effects in molecules \& polymers, Wiley-Interscience, New York, 1991; (b) M. J. Cho, D. H. Choi, P. A. Sullivan, A. J. P. Akelaitis and L. R. Dalton, Prog. Polym. Sci., 2008, 33, 1013-1058.

9 Thèse EPFL nr 2414 (2001). Rinuy, Juliette: Nonlinear optics of proteins at liquid interfaces and of DNA oligonucleotides in liquid phase.

10 M. G. Kuzyk, J. Pérez-Moreno and S. Shafei, Phys. Rep., 2013, 529, 297-398.

11 M. Verswyvel, F. Monnaie and G. Koeckelberghs, Macromolecules, 2011, 44, 8489-8498.

12 G. Olbrechts, R. Strobbe, K. Clays and A. Persoons, Rev. Sci. Instrum., 1998, 69, 2233-2241.

13 B. J. Orr and J. F. Ward, Mol. Phys., 1971, 20, 513.

14 (a) C. B. Gorman and S. R. Marder, Proc. Natl. Acad. Sci. U. S. A., 1993, 90, 11297-11301; (b) J. L. Oudar and D. S. Chemla, J. Chem. Phys., 1977, 66, 2664; (c) J. Zyss and J. L. Oudar, Phys. Rev. A, 1982, 26, 2016; (d) J. L. Brdas, F. Meyers, B. M. Pierce and J. Zyss, J. Am. Chem. Soc., 1992, 114, 4928.

15 S. R. Bhatta, Y. Y. Yimer, M. Tsige and D. S. Perry, Comput. Theor. Chem., 2012, 995, 36-42.

16 (a) G. M. Heffner and D. S. Person, Macromolecules, 1991, 24, 6295; (b) P. V. Shibaev, K. Schaumburg, T. Bjornholm and K. Norgaard, Synth. Met., 1998, 97, 97-104; (c) B. McCulloch, V. Ho, M. Hoarfrost, C. Stanley, C. Do, W. T. Heller and R. A. Segalman, Macromolecules, 2013, 46, 1899-1907.

17 J. Waite and M. G. Papadopoulos, J. Phys. Chem., 1990, 94, 6244-6249.

18 L.-T. Cheng, W. Tam, S. R. Marder, A. E. Stiegman, G. Rikken and C. W. Spangler, J. Phys. Chem., 1991, 95, 10643.

19 (a) B. Coe, J. A. Harris, I. Asselberghs, K. Clays, G. Olbrechts, A. Persoons, J. T. Hupp, R. C. Johnson, S. J. Coles, M. B. Hursthouse and K. Nakatani, Adv. Funct. Mater., 2002, 12, 110-116; (b) J. PérezMoreno, Y. Zhao, K. Clays and M. Kuzyk, Opt. Lett., 2007, 32, 59-61.

20 (a) C. L. Gettinger, A. J. Heeger, J. M. Drake and D. J. Pine, J. Chem. Phys., 1994, 101, 1673-1678; (b) R. R. Tykwinski, U. Gubler, R. E. Martin, C. Bosshard and P. Gu, J. Phys. Chem. B, 1998, 102, 4451-4465; (c) J. Hou, M.-H. Park, S. Zhang, Y. Yao, L.-M. Chen, J.-H. Li and Y. Yang, Macromolecules, 2008, 41, 6012-6018.

21 (a) M. Chang, D. Choi, B. Fu and E. Reichmanis, ACS Nano, 2013, 7, 5402-5413; (b) A. R. Aiyar, J.-I. Hong, R. Nambiar, D. M. Collard and E. Reichmanis, Adv. Funct. Mater., 2011, 21, 2652-2659. 